\title{
The properties of plain satin-like PLA/silk mixed fabrics
}

\begin{abstract}
The beautiful appearance and good properties make pure silk fabric one of the most popular fabrics. PLA (poly lactic acid) is a degradable Eco-friendly material which is made from corn or other crops, it also can be used in the textile engineering, and the PLA filament has good mechanical properties, such as high break elongation rate and breaking strength. It is a new task to combine these two types of textile materials for better use. This issue selected silk and PLA filament as warp and weft, 8 kinds of PLA/silk mixed fabrics were designed and weaved. In order to compare and analyze those fabrics, tests focusing on fabric properties, such as gas permeability, moisturepenetrability, wrinkle recovery ability and anti-pilling resistance, were carried out, through the comparison, it was found that PLA/silk mixed fabrics had excellent wearability, their moisture-penetrability were good, they did well in anti-pilling resistance although the air permeability and wrinkle recovery ability were no better than pure silk fabric.
\end{abstract}

Volume I Issue I - 2017

\author{
Ya-nan Zhu,' Zhi-juan Pan',2 \\ 'College of Textile and Clothing Engineering, Soochow \\ University, China \\ ${ }^{2}$ National Engineering Laboratory for Modern Silk, Soochow \\ University, China
}

Correspondence: Zhi-juan Pan, Professor, National Engineering Laboratory for Modern Silk, Soochow University, Suzhou, China, Tel+86 I362 5273 222,

Email zhjpan@suda.edu.cn

Received: November 22, 2016 | Published: February 13, 2017

\section{Introduction}

Poly lactic acid (PLA) is one of the most popular materials in recent years; it comes from natural crops which are full of starch. PLA is environmental friendly because it can be natural degraded into $\mathrm{CO} 2$ and $\mathrm{H} 2 \mathrm{O}$. PLA can be produced by lots of ways, especially by melt spinning. ${ }^{1}$ PLA can be spun into short fiber or filament, no matter pure spun or spun with other fibers. Nowadays, PLA is mainly produced by Cargill DOW Company, Chronopal Ltd. Co SHIMADZU and Mitsui Chemical. ${ }^{2}$ PLA filament is glossy and elastic with good mechanical properties such as high break elongation rate and good breaking strength, ${ }^{3}$ its high crystalline and orientation degree make it easier for dying and post processed, ${ }^{4}$ however, the high thermal shrinkage rate also exists. It will influence the post process for PLA filament.

Great amount of researches are based on PLA fiber properties, compared to this, studies on fabric design and fabric properties have a bright prospect for deeper research. Some Universities have already carried out researches on blended or mixed fabrics using PLA with other fibers. Xu Juan from Donghua University studied thermo stability and acid/alkali resistance of PLA fiber; researcher found that the PLA fiber's wet-hot resistance is poorer than its dryhot resistance. The hand feeling of PLA would turn harden when hot wet-air temperature is more than $100^{\circ} \mathrm{C} .{ }^{5}$ Liu Peng from Zhe Jiang
Sci-Tech University studied PLA filament properties, and PLA/wool, PLA/cotton; PLA/silk mixed fabrics were researched in his issue. ${ }^{6}$ Results show that PLA fabric has good wearing properties, it is soft and glossy, PLA can reduce the disadvantages which caused by the single material which was used in fabric. ${ }^{7}$ Based on the parameters of pure silk fabric, this research designed and weaved several mixed fabrics using PLA filament and pure silk. In order to compare those mixed fabrics with pure silk one, studies were focused on wear-ability including air permeability, moisture-penetrability, wrinkle recovery ability and anti-piling resistance. Through those tests, features of this kind of plain satin-like fabrics can be figured out.

\section{Materials and methods}

\section{Mechanical properties and thermal shrinkage proper- ties of PLA filament}

Instron5967 Material Testing Machine was used for testing the breaking strength, breaking strain and initial modulus of 50dtex/36f and 75dtex/48f PLA filaments. According to GB/T 6505-2008 "Test methods of thermal shrinkage properties for chemical fibers", tests for thermal shrinkage rate in wet and dry atmosphere were introduced, ${ }^{8}$ results of PLA filament's thermal shrinkage rate were shown in Table 1.

Table I Mechanical properties and thermal shrinkage properties of PLA filament

\section{Initial module (cN/ dtex)}

Breaking strain (\%)

\section{Breaking strength (cN/ dtex)}

Boiling water

shrinkage rate (\%)
Drying shrinkage rate

(\%) 50dtex PLA

$62.88 \pm 8.64$
$23.29 \pm 4.25$
$5.08 \pm 1.11$

13.49 


\section{The design and weaving of fabrics}

Depending on mechanical properties of PLA filament and regular specification parameters of plain satin silk, several kinds of fabrics were designed; parameters are showed in Table $2 .{ }^{10}$

In order to reduce the problems which may occur during weaving process, PLA filament needs to be starched before using as warp, sizing agent is mainly made from modified starch and PVA, temperature of sizing box is $55^{\circ} \mathrm{C}$, drying temperature is about $50^{\circ} \mathrm{C}$. After sizing, PLA filament will have better mechanical properties, which will be beneficial to PLA filament in later weaving process. ${ }^{11}$

Post processing of fabrics and finished product parameters

Post process after weaving is necessary for degumming, procedures were as following: Firstly, soak fabrics into warm water, then boil fabrics in refine solution which was the combination of refining agent, Table 2 Specification parameters of satin fabrics sodium silicate, sodium carbonate and hydrogen peroxide. Keep the bath ratio at 1:40. After boiling, there were 4 washing steps for 15 minutes each, the respectively water temperature were $85^{\circ} \mathrm{C}, 40^{\circ} \mathrm{C}$ and normal room temperature. Drying and ironing those fabrics after degumming. ${ }^{12}$

Product parameters are shown in Table 3; discrepancies exist among different samples because of the shrinkage rate of PLA filament and weaving shrinkage rate.

\section{Wear-ability tests of fabrics}

Air permeability: According to GB/T 5453-1997 "Textile-Determination of the permeability of fabrics to air". Air permeability was measured by M021 Machine, which had different rounded test areas and air pressure of $100 \mathrm{~Pa}$. Samples were all in length of 1 meter at least. Each sample was tested for 10 times, and then got the average values and standard deviations.

\begin{tabular}{|c|c|c|c|c|c|}
\hline \multirow[t]{2}{*}{ No. } & \multirow[t]{2}{*}{ Warp } & \multirow[t]{2}{*}{ Weft } & \multicolumn{2}{|c|}{ Designed density $(/ 10 \mathrm{~cm})$} & \multirow[t]{2}{*}{ Structure } \\
\hline & & & warp & weft & \\
\hline I & 3/22.2/24.4dtex2.5T silk & 50dtex/36f PLA & 720 & 500 & Five- heddle satin \\
\hline 2 & 3/22.2/24.4dtex2.5T silk & 75dtex/48f PLA & 720 & 500 & \\
\hline 3 & 3/22.2/24.4dtex2.5T silk & 75dtex/48f PLA & 720 & 400 & \\
\hline 4 & 3/22.2/24.4dtex2.5T silk & 4/22.2/24.4dtex2.5T silk & 720 & 500 & \\
\hline 5 & 75dtex/36f PLA & 3/22.2/24.4dtex2.5T silk & 720 & 400 & \\
\hline 6 & 75dtex/36f PLA & 3/22.2/24.4dtex2.5T silk & 720 & 500 & \\
\hline 7 & 75dtex/36f PLA & 4/22.2/24.4dtex2.5T silk & 720 & 500 & \\
\hline 8 & 75dtex/36f PLA & 5/22.2/24.4dtex2.5T silk & 720 & 500 & \\
\hline
\end{tabular}

Table 3 Parameters of finished product fabrics

\begin{tabular}{|c|c|c|c|c|c|c|c|c|}
\hline \multirow[t]{2}{*}{ No. } & \multirow[t]{2}{*}{ Warp } & \multirow[t]{2}{*}{ Weft } & \multicolumn{2}{|c|}{$\begin{array}{l}\text { Designed } \\
\text { density }(/ 10 \mathrm{~cm})\end{array}$} & \multicolumn{2}{|c|}{$\begin{array}{l}\text { Product } \\
\text { density }(/ 10 \mathrm{~cm})\end{array}$} & \multirow[t]{2}{*}{$\begin{array}{l}\text { Cover } \\
\text { factor }\end{array}$} & \multirow[t]{2}{*}{ Structure } \\
\hline & & & warp & weft & warp & weft & & \\
\hline 1 & 3/22.2/24.4dtex2.5T silk & 50dtex/36f PLA & 720 & 500 & 887 & 503 & 0.934 & $\begin{array}{l}\text { Five- heddle } \\
\text { satin }\end{array}$ \\
\hline 2 & 3/22.2/24.4dtex2.5T silk & 75dtex/48f PLA & 720 & 500 & 878 & 518 & $0.94 I$ & \\
\hline 3 & 3/22.2/24.4dtex2.5T silk & 75dtex/48f PLA & 720 & 400 & 828 & 403 & 0.897 & \\
\hline 4 & 3/22.2/24.4dtex2.5T silk & $\begin{array}{l}\text { 4/22.2/24.4dtex2.5T } \\
\text { silk }\end{array}$ & 720 & 500 & 753 & 508 & 0.892 & \\
\hline 5 & 75dtex/36f PLA & $\begin{array}{l}\text { 3/22.2/24.4dtex2.5T } \\
\text { silk }\end{array}$ & 720 & 400 & 743 & 453 & 0.854 & \\
\hline 6 & 75dtex/36f PLA & $\begin{array}{l}\text { 3/22.2/24.4dtex2.5T } \\
\text { silk }\end{array}$ & 720 & 500 & 748 & 568 & 0.887 & \\
\hline 7 & 75dtex/36f PLA & $\begin{array}{l}4 / 22.2 / 24.4 \mathrm{dte} 2.5 \mathrm{~T} \\
\text { silk }\end{array}$ & 720 & 500 & 743 & 563 & 0.907 & \\
\hline 8 & 75dtex/36f PLA & $\begin{array}{l}\text { 5/22.2/24.4dtex2.5T } \\
\text { silk }\end{array}$ & 720 & 500 & 758 & 528 & 0.92 & \\
\hline
\end{tabular}


Moisture-penetrability: According to GB/T 12704.2-2009 "Textile-test methods of water vapor transmission of fabrics-Part 2: water method", Textile test Fx3150 machine was used, fabrics were cut into circles of $7 \mathrm{~cm}$ diameters, 3 pieces of rounded samples were prepared for each fabric. Fixed those samples on soak cups which had $34 \mathrm{~mL}$ distilled water in them. Water-vapor transmission rate was tested after setting samples into the machine, and then got the average values.

Wrinkle recovery ability: According to GB/T 3819-1997 "Textile fabrics- Determination of the recovery from creasing of a folded specimen by measuring the angle of recovery", there were 5 samples prepared respectively for both warp and weft directions of each fabric, the pressure of measurement was $10 \pm 0.05 \mathrm{~N}$, time for quick recovery was $15 \pm 1 \mathrm{~s}$, time for slow recovery was $5 \mathrm{~min} \pm 5 \mathrm{~s}$. Each sample was pressed by the weight for 5 minutes, and then removed the weight, recovery angle in $15 \pm 1 \mathrm{~s}$ was defined as quick recovery angle, and recovery angle in $5 \min \pm 5 \mathrm{~s}$ was defined as slow recovery angle. Then got the average values for both quick and slow recovery angles in warp and weft directions. At last, each fabric's quick recovery angles in warp and weft direction were added together for defining as fabric's quick recovery ability. Slow recovery ability was gotten by the same way.

Anti-pilling resistance: According to GB/T 4802.2-2008 "Textile-Determination of the abrasion resistance of fabrics by the Martindale method", YG401G machine which took wool fabric as pilling material was used. Samples were fixed on pilling table; each sample was rubbed by wool fabric for 2000 times in prescribed track, then they were taken down from the machine after rubbing. Pilling rates were assessed in rating box according to the surface of each fabric.

\section{Results and discussion}

Fabrics in this paper were divided into 2 series, Series 1 includes plain satin-like PLA/silk mixed fabrics which used silk as warp (fabric 1-3) and pure silk fabric (fabric 4). Series 2 includes mixed fabrics which used PLA as warp (fabric 5-8). Discussion was primarily based on series 1 and 2. Results for wear ability of two Series were showed in Figures1-4.

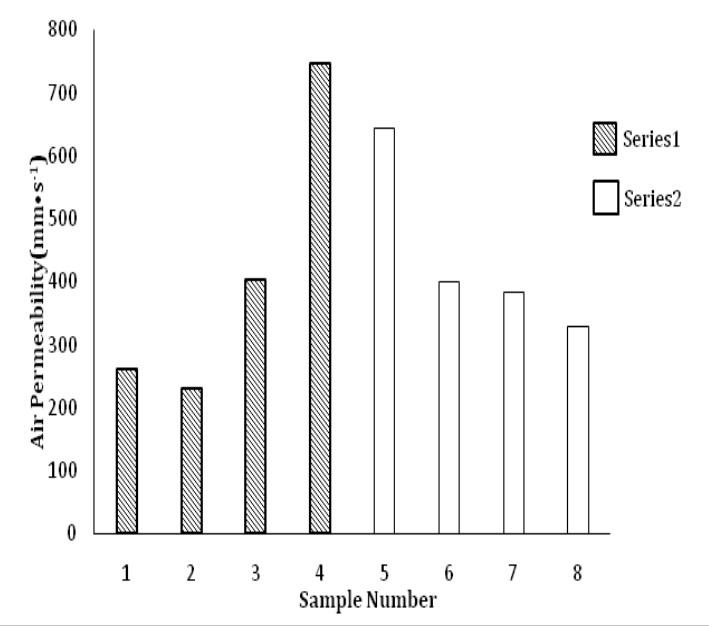

Figure I Air permeability of fabrics.

\section{Air permeability}

Air permeability mainly depends on fabric's tightness and structure. The looser, the easier for air to transfer. Comparing data in
Table 3 and Figure 1. Pure silk fabric had the highest air perm in series 1 , fabric 3 had the lower air perm compared to fabric 4 , which had the similar cover factor with it. It indicated that PLA filament could weaken the air permeability of fabrics. In series 2 , the increase of weft density and cover factor will reduce air perm. And it was obviously that fabric 5 had the highest air perm in series 2, according to Table 3, fabric 5 had the lowest cover factor, and its air permeability was the second-highest, it means although PLA filament will reduce the air perm, lower fabric cover factor is a good way to enhance this ability up to the level of pure silk fabric.

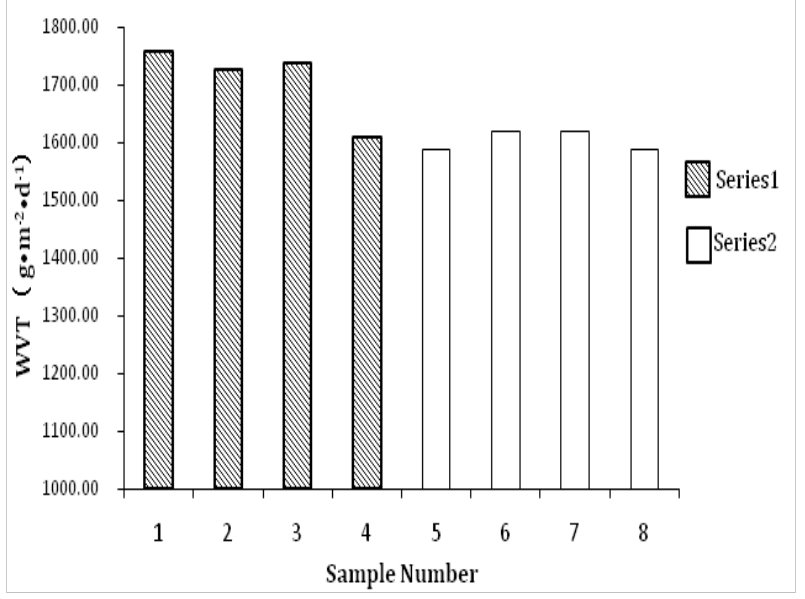

Figure 2 Moisture-penetrability of fabrics.

\section{Moisture-penetrability}

Moisture-penetrability is effected by water-absorbing quality of yarns and the tightness of fabric, water can transfer through the gaps among fiber and yarns in the fabric or connection points among yarns, although tighter fabric would have more connection points, the water transferred through them were much fewer than those transferred through the gaps among fiber and yarns in the fabric, so cover factor is still one of the most important factors of moisture-penetrability. Based on data in Table 3 \& Figure 2, all of the mixed fabrics had higher water-vapor transmission rate than pure silk fabric in series 1 , fabric 3 with the similar cover factor to fabric 4 had the higher watervapor transmission rate, it indicated that PLA filament would improve fabric moisture-penetrability, this was attribute to its special structure, there were many irregularly holes on the surface of PLA filament, and its outside structure was tighter than inside, this made it easier for PLA filament to absorb water as capillary effect exists. ${ }^{13}$ Comparing two series, series 1 had better moisture-penetrability than series 2, it means when using PLA filament as weft in PLA/silk mixed fabric, the combination of silk and PLA would have the best performance in this ability.

\section{Wrinkle recovery ability}

Wrinkle recovery ability depends on fibers' quality, yarns' elastic recovery ability and fabric structure. Figure 3 showed fabrics' wrinkle recovery angles. In series 1 , fabric 2 had the highest cover factor, and its recovery angle was the lowest. Almost all of wrinkle recovery angles of mixed fabrics were lower than fabric 4 . Fabric 3, which had the similar cover factor with fabric 4 also did worse in this ability, it indicated that PLA filament would affect fabric wrinkle recovery ability apparently. PLA filament had good elastic recovery ability, but high temperature and wet atmosphere made PLA macro molecules 
regrouped during the degumming process, which led to an increase of crystalline, then it would be difficult for PLA fabric to recover while getting external force. ${ }^{3}$ In series 2 , wrinkle recovery angle decreased with the increase of cover factor, however, fabric 8 did well although it had the highest cover factor, it was due to its thickness, weft of fabric 8 was thicker than other three fabrics, and it would be easier for the thicker one to recover after external force.

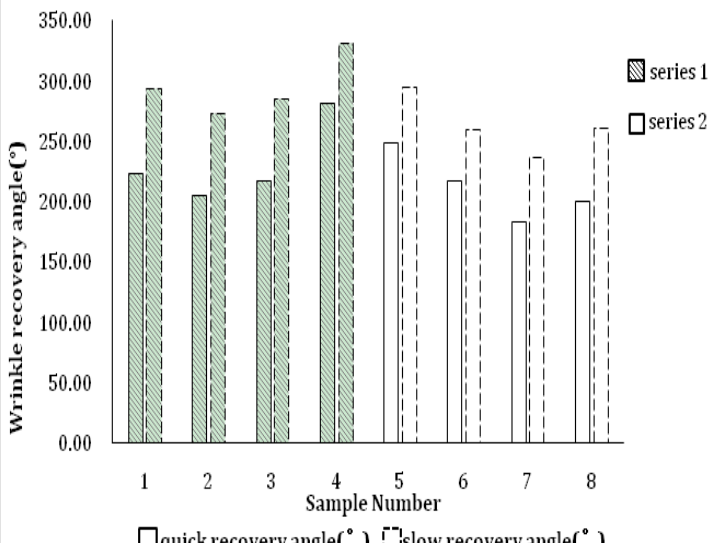

Figure 3 wrinkle recovery ability of fabrics.

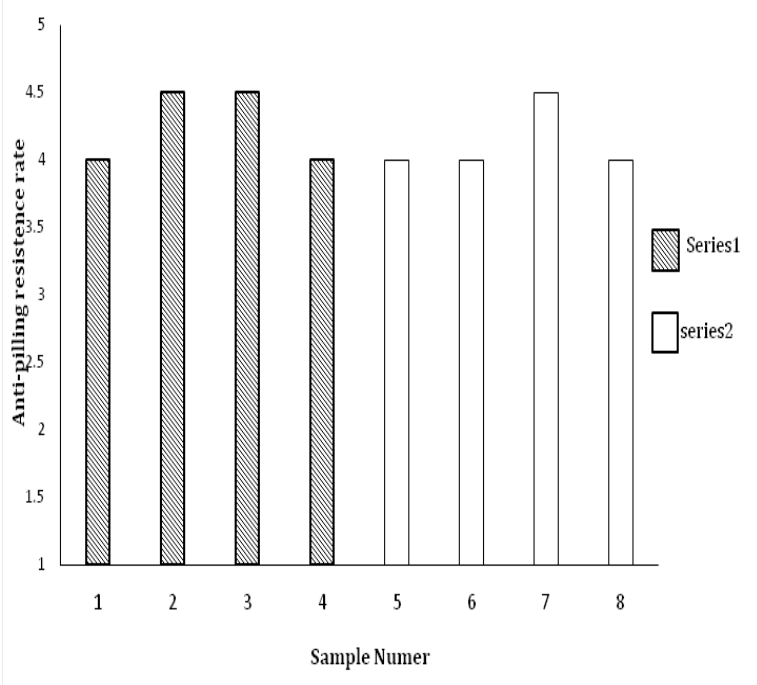

Figure 4 Anti-pilling resistance ability of fabrics.

\section{Anti-pilling resistance ability}

Anti-pilling resistance ability is associated with yarn quality and fabric structure. The fabrics with good anti-pilling ability not only refer to fabrics that infrequently get fuzz balls after pilling but also fabrics which fuzz balls could easily fall from surface. Comparing fabric 1-8, all of the mixed fabrics did not perform worse than fabric 4. Fabric 3, which had the lowest cover factor was even better than others, it demonstrated that PLA filament could enhance the antipilling resistance ability when mix weaved with silk. Although PLA filament had higher elastic recovery ability than silk, the high thermal shrinkage rate led to the change of PLA internal structure, chain folding and recrystallization harden the filament, so it would be difficult for fuzz balls attaching to the fabric surface.

\section{Conclusion}

In this issue, $3 / 22.2 / 24.4 \mathrm{dtex} 2.5 \mathrm{~T}$ silk and two types of PLA filaments (50dtex/36f PLA and 75dtex/48f PLA) were used as warp or weft, 7 kinds of plain satin-like mixed fabrics with different parameters and one piece of pure silk fabric were weaved. After comparing their properties of wear-ability, it was found that PLA filament could enhance fabric moisture penetrability and anti-pilling resistance ability, but lower fabric air permeability and wrinkle recovery ability.

Among these fabrics, fabric 3 performed the best in these properties, it's wonderful moisture penetrability and anti-pilling resistance ability demonstrated the fine combination of silk and PLA filament, the parameters of this match could be applied in real production.

During production, lower fabric cover factor and using PLA filament as weft will maximize the advantages of silk and PLA.

\section{Acknowledgments}

None.

\section{Conflict of interest}

Author declares there is no conflict of interest in publishing the article.

\section{References}

1. You Xinqiang. Research on Synthesis Process of Poly lactic Acid. Graduation thesis of Chongqing University. China; 2012. p. 1-10

2. Chen Jida, Hu Chengbo, Zhang Yonghong. Introduction to the research progress of lactide. Journal of Tissue Engineering and Reconstructive Surgery. 2006;2006(4):185.

3. Zhang Ruiyun. Research on PLA covering yarn properties and product development. Graduation thesis of Donghua University: China; 2013. p. $1-54$

4. Zhao Yongxia, Dong Kuiyong. The truth clarification of new type fiber (V) PLA fiber, China. Textile Leader. 2010;(3):55-56

5. Xu Juan, Guo Jiansheng. Acid-base resistance property of PLA fiber Synthetic Fiber in China. 2011;40(11):25-29.

6. Liu Peng. Research on Properties of PLA filament and mixed fabric. Zhejiang Sci-Tech University: China; 2011. p. 9-38

7. Liu Peng, Feng Zhaoxing. Industrial development tendency of PLA fabric. Modern Commerce. 2010;(36):171.

8. Professional experiment tutorial of Textile engineering. Soochow University: China; 2005.

9. Unified specification table for exported silk satin. The China National Silk Industry Corporation: China; 1990.

10. Yang Yuan, Zhou Jianping, Li Yonggui, et al. Performances research of PLA fiber and it's blended fabric. Shanghai Textile Science \& Technology. 2008;2008(2):54-56.

11. Xu Weihong, Xue Rong. Sizing process of PTT fiber blended with cotton. Jiangsu Textile. 2007;1(6):29.

12. Zhu Simin. Design and Development of New Chemical Fiber/Silk Multicomponent Compound Fabric. Graduation thesis of Soochow University: china; 2014. p. 1-65.

13. Jiang Quanguang, Li Yabin. Research tendency of reproducible PLA fiber. Tianjin Textile Science \& Technology. 2012;(2):1-4. 